\title{
Astrobiology: Towards an Understanding of the Emergence of Life in the Universe
}

\author{
Antonio Lazcano \\ Facultad de Ciencias, UNAM, Apdo. Postal 70-407 Cd. Universitaria, \\ 04510 México, Mexico
}

\section{Introduction}

Long before the idea of spontaneous generation was incorporated by JeanBaptiste de Lamarck into evolutionary biology to explain the first emergence of life, the possibility that other planets were inhabited had been discussed, sometimes in considerable detail, by scientists and philosophers alike (Lazcano 2001). More often than not, these were speculations that rested on the idea of a uniform universe but with little or no empirical basis. Today our approaches to the issue of life in the Universe have changed dramatically; neither the formation of planets nor the origin of life are seen as the result of inscrutable random events, but rather as natural outcomes of evolutionary events. The interconnection between these two processes is evident: understanding the formation of planets has major implications for our understanding of the early terrestrial environment, and therefore for the origin of living systems.

Although it is tempting to assume that the emergence of life is an unavoidable process that may be continuously taking place throughout the Universe, it is still to be shown that it exists (or has existed) in places other than the Earth. With the exception of Mars and some speculations on Europa, prospects for life in our own Solar System have diminished. Although there is evidence the early Martian environment was milder and may have been similar to the primitive Earth, today its surface is a deep-frozen dessert, constantly bathed by short-wavelength ultraviolet radiation. This highly oxidizing environment has rendered any hypothetical biosphere extinct or has limited it to a few restricted underground niches well below the surface, where brine aquifers appear to be present. Indeed, the balance of evidence suggests that life in our planetary system is confined to our own planet. As shown by the debates sparked by the announcement that the Allan Hills 84001 meteorite included traces of ancient Martian life (McKay et al. 1996), we also lack a well-defined consensus regarding the criteria by which we could rapidly recognize evidence of extraterrestrial biological activity.

Recognition that meteorite impacts may have led to an intense exchange of rocky ejecta between the inner planets during the early phases of the Solar System has led some to discuss the possibility that life on our planet may have an ultimate Martian origin (Nisbet \& Sleep 2001). It is somewhat amusing to see that discussions on panspermia, i.e., the transfer of organisms from one planet to another, are periodically resurrected without providing detailed explanations of the ultimate mechanisms which may have led to the appearance of life in extraterrestrial habitable environments. It is true that the high UV-resistance of 
different prokaryotic species at the low temperatures of deep space, the likelihood of artificial or directed transport of microorganisms by probes sent to other bodies in the solar system, and the recognition of the Martian origin of some meteorites have given additional support to the panspermia hypothesis (Horneck 1998). However, this only shifts the problem to a different location, and most researchers prefer to study the origin of life within the historical framework of an evolutionary analysis that assumes that it took place on Earth.

\section{The Antiquity of life on Earth}

As shown by the recent debates, determination of the biological origin of what have been considered the earliest traces of life can be a rather contentious issue (van Zullen et al. 2002). The geological record of the early Archean is even scarcer, and most of the rocks which have been preserved have been metamorphosed to a considerable extent. However, there is evidence that life emerged on Earth as soon as it was possible to do so. The biological origin of the microstructures interpreted as cyanobacterial remnants in the $3.5 \times 10^{9}$ year old Apex sediments of the Australian Warrawoona formation (Schopf 1993) have been challenged (Brasier et al. 2002). Although it is possible that the ultimate origin of some of these structures may be explained by non-biological processes, others are clearly endowed with the diagnostic features of a microbial community associated with a seafloor hydrothermal system (Van Kranendonk 2002).

Thus, although traditionally it had been assumed that the origin and early evolution of life involved several billions of years (Oparin 1938; Huang 1959; Cloud 1968; Dickerson 1978), such views are no longer tenable. While it is true it is not possible to assign a precise chronology to the appearance of life, in the last few years estimates of the available time for this to occur have been considerable reduced. The planet is generally thought to have remained molten for several hundred million years after its formation $4.6 \times 10^{9}$ years ago (Wetherill 1990), and late accretion impacts may have boiled-off the oceans and destroyed all life in the planets as late as $3.8 \times 10^{9}$ years ago (Sleep et al. 1989). However, there is compelling paleontological evidence that highly diverse microbial communities were thriving during the early and middle Archean (Nisbet \& Sleep 2001; Schopf et al. 2002; Van Kranendonk 2002).

Such rapid development speaks for the relatively short timescale required for the origin and early evolution of life on Earth, and suggests that the critical factor may have been the presence of liquid water, which became possible once the planet's surface cooled down. But as of today we do not know whether the emergence of terrestrial life was a highly probable event because the role of chance in chemical evolution was strongly constrained by environmental conditions and the properties inherent to prebiotic chemical systems, or if we are the unique outcome of an unlikely series of random events.

\section{The Molecular Fossil Record}

The awareness that genes and genomes are extraordinarily rich historical documents from which a wealth of evolutionary information can be retrieved has widened the range of phylogenetic studies to previously unsuspected heights. 
The development of efficient nucleic acid sequencing techniques, which now allows the rapid sequencing of complete cellular genomes, combined with the simultaneus and independent blossoming of computer science, has led not only to an explosive growth of databases and new sophisticated tools for their exploitation, but also to the recognition that different macromolecules may be uniquely suited as molecular chronometers in the construction of nearly universal phylogenies.

Molecular cladistics may provide clues to some very early stages of biological evolution. However, it is difficult to see how the applicability of this approach can be extended beyond a threshold that corresponds to a period of cellular evolution in which protein biosynthesis was already in operation. Older stages are not yet amenable to molecular phylogenetic analysis. Although there have been considerable advances in the understanding of chemical processes that may have taken place before the emergence of the first living systems, life's beginnings are still shrouded in mystery. A cladistic approach to this problem is not feasible, since all possible intermediates that may have once existed have long since vanished. The temptation to do otherwise is best resisted. Given the huge gap existing in current descriptions of the evolutionary transition between the prebiotic synthesis of biochemical compounds and the last common ancestor of all extant living beings (Lazcano 1994), it is naive to attempt to describe the origin of life and the nature of the first living systems from the available rooted phylogenetic trees.

Nevertheless, there have been several attempts to use macromolecular data to support claims on the hyperthermophily of the first living organisms and the idea of a hot origin of life. The examination of the prokaryotic branches of unrooted rRNA trees had already suggested that the ancestors of both eubacteria and archaebacteria were extreme thermophiles, i.e., organisms that grow optimally at temperatures in the range $90^{\circ} \mathrm{C}$ and above (Achenbach-Richter et al. 1987). Rooted universal phylogenies appear to confirm this possibility, since heat-loving bacteria occupy short branches in the basal portion of molecular cladograms (Stetter 1994).

Such correlation between hyperthermophily and primitiveness has led support to the idea that heat-loving lifestyles are relics from early Archean hightemperature regimes that may have resulted from a severe impact regime (Sleep et al. 1989). It has also been interpreted as evidence of a high temperature origin of life, which according to these hypotheses took place in extreme environments such as those found today in deep-sea vents (Holm 1992) or in other sites in which mineral surfaces may have fuelled the appearance of primordial chemoautolithotrophic biological systems (Wächtershäuser 1990).

Although no mesophilic organisms older than heat-loving bacteria have been discovered, it is possible that hyperthermophily is a secondary adaptation that evolved in early geological times (Sleep et al. 1989; Confalonieri et al. 1993; Lazcano 1993). Such possibility is in fact strongly supported by the phylogenetic analysis of the $\mathrm{G}+\mathrm{C}$ content of rRNA genes, which suggest that the last common ancestor was not a hyperthermophilic organism (Galtier et al. 1999). In fact, hyperthermophiles not only share the same basic features of the molecular machinery of all other forms of life; they also require a number of specific biochemical adaptations. Any theory on the hot origin of life must address the question 
of how such traits, or their evolutionary precedessors, arose spontaneously in the prebiotic environment. Such adaptations may include histone-like proteins, RNA modificating enzymes, and reverse gyrase, a peculiar ATP-dependent enzyme that twists DNA into a positive supercoiled conformation (Confalonieri et al. 1993). Clues to the origin of hyperthermophily may be hidden in this list. Thus, although the antiquity of hyperthermophiles appears to be well established, there is no evidence that they have a primitive molecular genetic apparatus. The most basic questions pertaining to the origin of life relate to much simpler replicating entities predating by a long series of evolutionary events the oldest recognizable heat-loving bacteria. Why hyperthermophiles are located at the base of universal trees is still an open question, but the possibility that adaptation to extreme environments is part of the evolutionary innovations that appeared in the trunk of the tree cannot be entirely dismissed.

\section{An Heterotrophic Origin of Life?}

It is unlikely that data on how life originated will be provided by the palaeontological record. There is no geological evidence of the environmental conditions on the Earth at the time of the origin of life, nor any fossil register of the evolutionary processes that preceded the appearance of the first cells. Direct information is lacking not only on the composition of the terrestrial atmosphere during the period of the origin of life, but also on the temperature, ocean $\mathrm{pH}$ values, and other general and local environmental conditions which may or may not have been important for the emergence of living systems. Given this situation, it is therefore not surprising that several alternative and even opposing theories have been proposed.

The idea of life as an emergent feature of Nature was widespread during the last century, but it was not until Oparin (1938) proposed that first living systems were heterotrophic microorganisms that resulted from the evolution of abiotically synthesized organic compounds and the formation of a self-sustaining supramolecular systems, that the study of the origin of life was transformed from a purely speculative discussion into a workable research program. Today scientific efforts in this field are not necessarily oriented towards the in vitro production of a living system, but rather towards the construction a coherent historical narrative by weaving together a large number of miscellaneous observational findings and experimental results.

The hypothesis of chemical evolution is supported not only by a number of laboratory simulations, but also by a wide range of astronomical observations and the analysis of samples of extraterrestrial material. These include the existence of organic molecules of potential prebiotic significance in interstellar clouds and cometary nuclei, and of small molecules of considerable biochemical importance that are present in carbonaceous chondrites. The copious array of amino acids, carboxylic acids, purines, pyrimidines, hydrocarbons, and other molecules which have been found in the $4.5 \times 10^{9}$ years-old Murchison meteorite and other carbonaceous chondrites gives considerable credibility to the idea that comparable syntheses took place in the primitive Earth (Or- et al. 1990; Becker et al. 2002). 
There is also strong experimental support for the idea of prebiotic formation of organic molecules. The first successful synthesis of biochemical compounds under plausible primordial conditions was accomplished by the action of electric discharges acting for a week over a mixture of $\mathrm{CH}_{4}, \mathrm{NH}_{3}, \mathrm{H}_{2} \mathrm{O}$, and $\mathrm{H}_{2}$, yielding a racemic mixture which included several proteinic and non-proteinic amino acids, as well as hydroxy acids, urea, and other organic molecules (Miller 1953). A few years later, Or- (1960) showed that adenine, a purinic compound that plays a central role in both genetic processes and biological energy utilization, was a major product of the non-enzymatic condensation of $\mathrm{HCN}$, which in turn is an abundant constituent of interstellar clouds and cometary nuclei. The potential role of HCN as a precursor in prebiotic chemistry has been further supported by experimental evidence showing that the hydrolytic products of its polymers include amino acids, purines, and orotic acid, an intermediate in the biosyntheses of uracil and cytosine, two major components of RNA, indicating that diverse biochemical compounds could had been formed simultaneously from simple reactants (Ferris et al. 1996). Laboratory syntheses under possible primitive conditions of other organic compounds of biochemical significance, such as tricarboxylic acids, alcohols, porphyrins, fatty acids, and a number of coenzymes has been reviewed elsewhere (Or- et al. 1990).

The above results suggest that the prebiotic soup must have been a bewildering organic chemical wonderland, but it could not include all the compounds or the molecular structures found today even in the most ancient extant forms of life - nor did the first cellcomplexity of the prebiotic environment, life could not have s spring completely assembled, like Frankestein's monster, from simple precursors present in the primitive oceans. The fact that a number of chemical constituents of contemporary forms of life can be synthesized non-enzymatically under laboratory conditions does not necessarily imply by itself that they were also essential for the origin of life, or that they were available in the primitive environment. Moreover, the lack of agreement on the chemical constituents of the primitive atmosphere has also led to major debates. Although it is generally accepted that free oxygen was absent, many planetologists favor the possibility that it consisted of much less-reduced gases such as $\mathrm{CO}_{2}, \mathrm{~N}_{2}$, and $\mathrm{H}_{2} \mathrm{O}$ (Kasting 1993), while prebiotic chemists prefer more reducing mixtures (Lazcano \& Miller 1996).

The correlation between the compounds which are produced in prebiotic simulations and those found in carbonaceous meteorites (Becker et al. 2002) is too striking to be fortuitous, and strongly supports the contention that such molecules were part of the chemical environment from which life evolved. However, the leap from biochemical monomers and small oligomers to living cells is enormous. There is a major gap between the current descriptions of the primitive soup and the appearance of non-enzymatic replication. Solving this issue is essential to our understanding of the origin of the biosphere: regardless of the chemical evolved in the absence of a genetic replicating mechanism insuring the maintenance, stability, and diversification of its basic components.

\section{The RNA World and the Origin of Replication}

How the ubiquitous nucleic acid-based genetic system of extant life originated is one of the major unsolved problems in contemporary biology. The discov- 
ery of catalytically active RNA molecules gave considerable credibility to prior suggestions of that the first living organisms were largely based on ribozymes an hypothetical stage called the RNA world (Gilbert 1986; Joyce 2002). This possibility is now widely accepted, but the chemical lability of RNA components suggests that this molecule was not a direct outcome of prebiotic evolution. It is unlikely that wriggling RNA molecules were floating in the primitive ocean, ready to be used as primordial genes. As reviewed elsewhere (Lazcano \& Miller 1996), from the chemical viewpoint the RNA world faces major problems, which include the origin of its ribose moiety, the rapid decomposition of this and other sugars under primitive conditions, and the availability of polyphosphates and phosphate esters, which are not prebiotic reagents.

The above difficulties have led to proposals of pre-RNA worlds, in which informational macromolecules with backbones different from those of extant nucleic acids may have also been endowed with catalytic activity, i.e., with phenotype and genotype also residing in the same molecules. The nature of the genetic polymers and the catalytic agents that may have preceded RNA is of course unknown. One interesting candidate are the so-called peptide nucleic acids, or PNAs, which are linear polymers in which the sugar-phosphate backbones of RNA and DNA are replaced by uncharged peptide-like backbones formed by achiral amino acid units linked by amide bond, to which the bases are covalently attached (Nielsen 1993). However attractive PNAs may be to some of us, the origin of non-enzymatic replication remains a major, unsolved problem. Nonetheless, new experimental models provide interesting insights. Enhancement of monomer concentration in experimental systems simulating a drying lagoon has achieved a successful surface-bound template polymerization up to 53 nucleotides (Ferris et al. 1996), and the chiroselective self-assembly of long homochiral oligomers of nucleic acid analogues from racemic mixtures of smaller chains into oligomers has been reported (Bolli et al. 1997).

There is evidence suggesting that replication may be a widespread phenomenon that includes chemical systems lacking the familiar nucleic acid structure. This possibility is supported by (a) a 32-residue alpha-helical peptide which can template and catalyze its own synthesis from activated smaller fragments under aqueous conditions (Lee et al. 1996); (b) a horseshoe-shaped product of the chemical reaction between an amino-adenosine and a complex aromatic ester, whose product enhances the formation of similar molecules in a non-aqueous solvent (Hong et al. 1992); and (c) synthetic micelles containing lithium hydroxyde and stabilized by an octanoid acid derivative, which swim in an organic solvent that acts as a substrate for the formation of additional micelles (Bachmann et al. 1992). While it is unlikely that these non-informational autocatalytic systems are ancestral to our own DNA-based cellular reproduction, their diversity suggests that chemical replicative systems may be much more widespread in Nature than previously thought.

\section{Final Remarks}

The basic tenet of the heterotrophic theory is that the maintenance and reproduction of the first living systems depended primarily on prebiotically synthesized organic molecules. There has been no shortage of discussion about how 
the formation of the primitive soup took place. However, it is likely that no single mechanism can account for the wide range of organic compounds that may have accumulated on the primitive Earth, and that the prebiotic soup was formed by contributions from endogenous syntheses in a reducing atmosphere, metal sulphide-mediated synthesis in deep-sea vents, and exogenous sources such as comets, meteorites and interplanetary dust. This ecclectic view does not beg the issue of the relative significance of the different sources of organic compounds -it simply recognizes the wide variety of potential sources of organic compounds, the raw material required for the emergence of life.

As discussed here, the existence of different abiotic mechanisms by which biochemical monomers can be synthesized under plausible prebiotic conditions is well-established. Of course, not all prebiotic pathways are equally efficient, but the wide range of experimental conditions under which organic compounds can be synthesized demonstrates that prebiotic syntheses of the building blocks of life are robust, i.e., the abiotic reactions leading to them do not take place under a narrow range defined by highly selective reaction conditions, but rather under a wide variety of experimental settings. Our ideas on the prebiotic synthesis of organic compounds are based largely on experiments in model systems. The robustness of this type of chemistry is supported by the occurrence of most of these biochemical compounds in the Murchison meteorite. So it becomes plausible, but not proven, that similar synthesis took place on the primitive Earth. For all the uncertainties surrounding the emergence of life, it appears to us that the formation of the prebiotic soup is one of the most firmly established events that took place in the primitive Earth.

Thus, if convincing processes can be demonstrated for the origin of life on Earth, then it is reasonable to conclude that life is the natural outcome of an evolutionary process, and that it may have appeared elsewhere in the universe. Although we do not know how the transition from the non-living to the living took place, most of the modern scenarios start out with relative simple organic molecules, now known to be widely distributed (Becker et al. 2002), which are readily synthesized, and hypothesized to undergo further evolutionary changes leading into self-maintaining, self-replicative systems from which the current DNA/protein-based biology resulted.

The distinguished American evolutionist George Gaylord Simpson (1973) once wrote that "exobiology is still a science without any data, therefore no science". The same, of course, could not be said today of astrobiology. The idea that life is the result of a rare chance event has been replaced by an evolutionary narrative, according to which biological systems are the outcome of a gradual but not necessarily slow process that begun with the abiotic synthesis of biochemical monomers and eventually led to self-sustaining, self-replicating systems capable of undergoing Darwinian evolution. There is no compelling reason to assume that such processes took place only on the Earth. The timescale for the origin and early evolution of life and the ease of formation of amino acids, purines, and other blochemical compounds under a relatively wide range of reducing conditions, together with the abundance of organic molecules throughout space, all speak for natural laws conductive to the emergence of life in extraterrestrial environments where similar conditions prevail. 
Yet, the role of historical contingency cannot be discounted. As the French philosopher Pascal once remarked, had Cleopatra's nose been different, the course of history may have changed. Precellular evolution was not a continuous, unbroken chain of progressive transformations steadily proceeding to the first living systems. Many prebiotic cul-de-sacs and false starts probably took place. While it may be true that the transition to life from non-living systems did not require a rather narrow set of environmental constrains, we cannot discount the possibility that even a slight modification of the primitive environment could have prevented the appearance of life on our planet. However unpalatable this conclusion may be, life may be a rare and even unique phenomenon in the Universe.

Acknowledgments. A. L. is an Associate of the NSCORT (NASA Specialized Center for Research and Training) in Exobiology at the University of California, San Diego. I am indebted to Professor Stanley L. Miller for many discussions on this subject, which have led to different publications in which these issues are discussed. Support from DGAPA-UNAM during a sabbatical leave of absence in the laboratory of Professor R. Amils (Universidad Aut-noma de Madrid) is gratefully acknowledged.

\section{References}

Achenbach-Richter, L., Gupta, R., Stetter., K. O., \& Woese, C. R. 1987, System. Appl. Microbiol., 9, 34

Bachmann, P. A., Luisi, P. L., \& Lang, J. 1992, Nature, 357, 57

Becker, L., et al. 2002, Rep. Prog. Phys., 65, R1

Bolli, M., Micura, R., \& Eschenmoser, A. 1997, Chemistry \& Biology, 4, 309

Brasier, M., et al. 2002, Nature, 416, 76

Cloud, P. E. 1968, Science, 160, 729

Confalonieri, F., Elie, C., Nadal, M., Bouthier de la Tour, C., Forterre, P., \& Duguet, M. 1993, Proc. Natl. Acad. Sci. USA, 90, 4753

Dickerson, R. E. 1978, Sci. Am., 239, 70

Ferris, J. P., Hill, A. R., Liu, R., \& Orgel, L. E. 1996, Nature, 381, 59-61

Galtier,, N., Tourasse, N., \& Gouy, M. 1999, Science, 283, 220

Gilbert, W. 1986, Nature, 319, 618

Holm, N. G., ed. 1992, in Marine Hydrothermal Systems and the Origin of Life (Dordrecht: Klüwer Academic Publ.), 242

Hong, J. 1., Feng, Q., Rotello, V., \& Rebek, J. Jr. 1992, Science, 255, 848

Horneck, G. 1998, in Trieste Conference on Chemical Evolution. V. Exobiology, matter, energy, and information in the origin and evolution of life in the Universe, ed. J. Chela Flores \& F. Raulin, (Dordrecht: Klüwer Academic Publishers), 205

Huang, S. S. 1959, Publ. Astron. Soc. Pacific, 71, 421

Joyce, G. F. 2002, Nature, 418, 214

Kasting, J. F. 1993, Science, 259, 920 
Lazcano, A. 1993, Science, 260, 1154

Lazcano, A. 1994, in Early Life on Earth, Nobel Symposium No. 84, ed. S. Bengtson, (New York: Columbia University Press), 60

Lazcano, A. 2001, in The search for life in the Universe, ed. F. Giovanelli, (Roma: Consiglio Nazionale delle Ricerche), 379

Lazcano, A., \& Miller, S. L. 1996, Cell, 85, 793

Lee, D. H., Granja, J. R., Martinez, J. A., Severin, K., \& Ghadri, M. R. 1996, Nature, 382, 525

McKay, D. S., et al. 1996, Science, 273, 924

Miller, S. L. 1953, Science, 117, 528

Nielsen, P. E. 1993, Origin Life Evol. Biosph., 23, 323

Nisbet, E. G., \& Sleep, N. H. 2001, Nature, 409, 1083

Oparin, A. I. 1938, The Origin of Life, (NewYork: MacMillan)

Or-, J. 1960, Biochem. Biophys. Res. Comm., 2, 407

Or-, J., Miller, S. L., \& Lazcano, A. 1990, Annu. Rev. Earth Planet. Sci., 18, 317

Schopf, J. W. 1993, Science, 260, 640

Schopf, J. W., Kudryavtsev, A. B., Agresti, D. G., Wdowiak, T. J., \& Czaja, A. D. 2002, Nature, 416, 73

Simpson, G. G. 1973, in Communication with Extraterrestrial Intelligence, ed. C. Sagan, (Cambridge: MIT Press), 362

Sleep, N. H., Zahnle, K. J., Kasting, J. F., \& Morowitz, H. J. 1989, Nature, 342, 139

Van Kranendonk, M. J. 2002, in Abstracts of the IAU Symposium 213 Bioastronomy 2002: Life among the Stars (Hamilton Island - Australia), 33

van Zullen, M. A., Lepland, A., \& Arrhenius, G. 2002, Nature, 418, 627

Wächtershäuser, G. 1990, Origins of Life Evol. Biosph., 20, 173

Wetherill, G. W. 1990, Annu. Rev. Earth Planet. Sci., 18, 205 


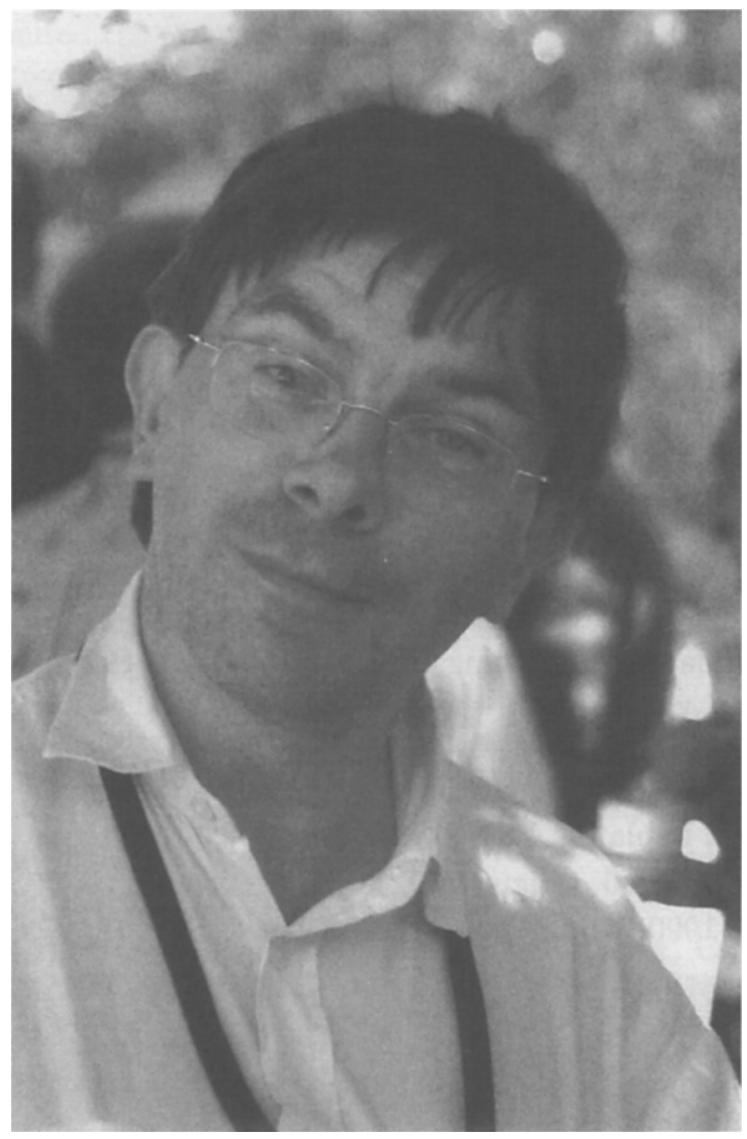

Simon Conway Morris (photo: Seth Shostak) 